\title{
Evaluation of Different 'Tensioning' of Apical Suspension in Women Undergoing Surgery for Prolapse and Urinary Incontinence
}

\author{
SEBASTIAN LUDWIG, SAMIRA GÖKTEPE, PETER MALLMANN and WOLFRAM JÄGER \\ Division of Urogynecology and Pelvic Reconstructive Surgery, Department of Obstetrics and Gynecology, \\ University Hospital Cologne and Medical Faculty, Cologne, Germany
}

\begin{abstract}
Background: According to the literature, restoration of pelvic organ prolapse also restores urinary continence. However, it is difficult to determine which exact surgical procedure actually led to urinary continence due to a lack of standardisation among these techniques. For example, an apical fixation is broadly defined in terms of type and shape of implanted material, anatomical fixation sides and tensioning. The aim of this study was to evaluate the effect of bilateral uterosacral ligament replacement with alloplastic tapes of defined lengths on symptoms of urinary incontinence. Patients and Methods: Patients with urinary incontinence and pelvic organ prolapse underwent an apical suspension. Thereby, both uterosacral ligaments (USL) were replaced by alloplastic structures of defined length. These alloplastic tapes had defined lengths in all patients of 9, 10, or $11 \mathrm{~cm}$ in length. They were sutured on both sides of the cervix, placed in the left and right peritoneal fold of the USL, and were sutured laterally to the prevertebral fascial layer of the sacral vertebra at the level of $S 2$. Furthermore, all patients received additional transobturator tape. Pelvic organ prolapse was classified according to the Baden-Walker system, and urinary incontinence symptoms according to validated questionnaires. Results: Four months after bilateral USL replacement, apical suspension was restored in all 31 patients. In addition, urinary continence was reestablished in 18 out of these 31 patients (58\%). The highest
\end{abstract}

This article is freely accessible online.

Correspondence to: Wolfram Jäger, MD, Ph.D., Department of Obstetrics and Gynecology, Division of Urogynecology and Pelvic Reconstructive Surgery, University Hospital Cologne and Medical Faculty, Kerpener Str. 34, 50931, Cologne, Germany. Tel: +49 22147897303, e-mail: wolframjaeger@gmx.de

Key Words: Bilateral apical fixation, integral theory, transobturator tape, urinary incontinence, uterosacral ligaments. continence rate was observed in patients in which both USL were replaced with alloplastic tapes of $9 \mathrm{~cm}$ in length. Conclusion: The findings indicated the importance of USL integrity for urinary continence. In particular, a defined length for both USL seems to be important for (re)establishing urinary continence.

In 1992, Ulmsten and Petros introduced the Integral Theory, which states that urinary incontinence (UI) in women is caused by diminishing anatomical support of the bladder base $(1,2)$. They hypothesised that both forms of UI, stress urinary incontinence (SUI) as well as urgency urinary incontinence (UUI), are caused by laxity of the anterior vaginal wall. This laxity of the anterior vaginal wall leads to the funnelling of the bladder neck and the activation of stretch receptors, triggering inappropriate micturition reflexes (3). The aetiology of this laxity of the anterior vaginal wall is under debate. Because the vagina is attached to the cervix of the uterus, any descent of the uterus will lead to reduced tension of the anterior vaginal wall. To treat UI, Ulmsten and Petros therefore proposed the tension of the anterior vaginal wall be 'tightened' by restoration of the uterosacral ligaments (USL) and pubo-urethral ligaments (PULs) (1).

Instead of performing a vaginal hysterectomy or unilateral sacropexy as usual, we considered changing our treatment of apical prolapse in patients with accompanying UI.

According to the Integral Theory, we replaced the USL and PUL in these patients. For the repair of the PUL, we used the established transobturator tape (TOT) technique according to Delorme et al. $(4,5)$. This procedure has been well described and involves the placement of the tape at a distance of approximately $4 \mathrm{~mm}$ below the urethra. The tape should not be tightened but be simply placed there 'tension-free'.

To the best of our knowledge, a standardised surgical technique for apical suspension (bilateral replacement of the USL) has not been published so far. Amundsen et al. proposed a duplication of the USL, thereby shortening the ligaments but without defined tensioning (6-8). 


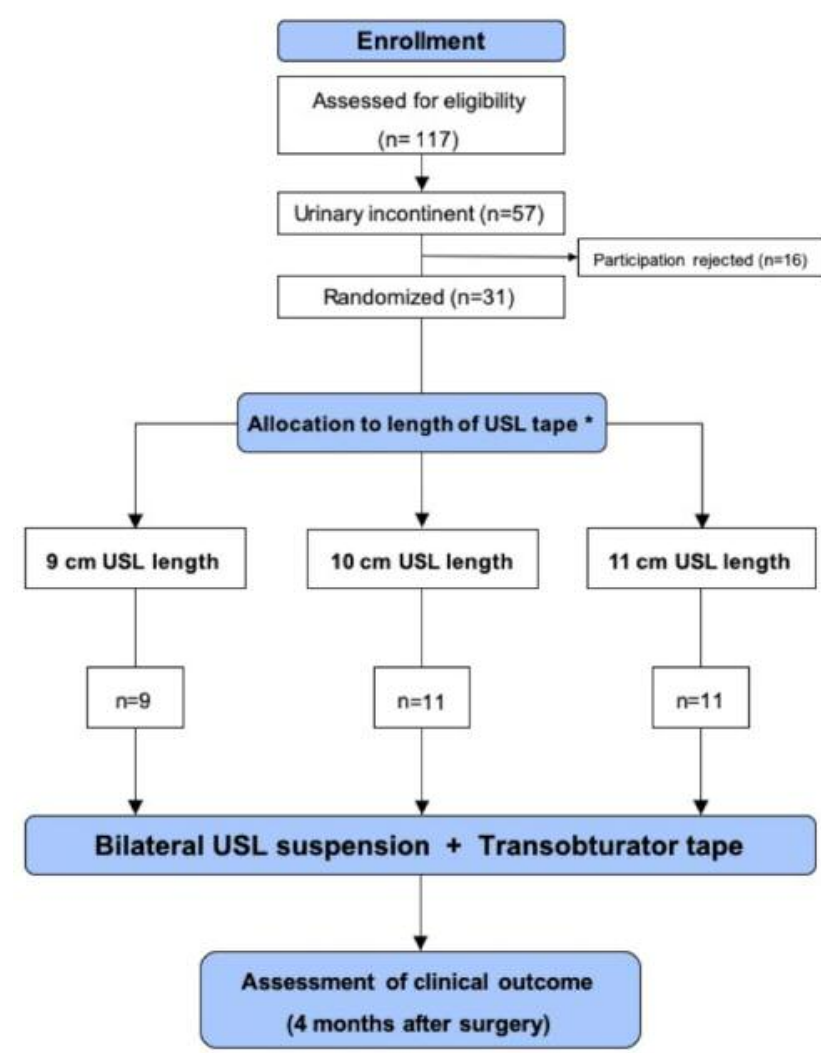

Figure 1. Flowchart of the 31 patients with prolapse of the uterus and urinary incontinence undergoing apical suspension and transobturator tape placement. *Both uterosacral ligaments (USL) were replaced by alloplastic tapes of defined length (either 9, 10, or $11 \mathrm{~cm}$ ).

To restore prolapse of the uterus, both USL were replaced by alloplastic structures ('tapes') of defined length and shape. This bilateral fixation between the cervix and sacral vertebra would definitely tension the vagina. The origin of the USL at the posterior cervix and lateral at the sacral vertebra in front of S2 have been well studied $(9,10)$. According to the literature, the length of USL vary up to $14 \mathrm{~cm}$ (11). However, these studies did not consider the patient's age or stage of prolapse in the supine body position.

In order to determine a 'physiological' length of USL replacement, we placed small alloplastic (polytetrafluoroethylene) tapes of different lengths $(9,10$, and $11 \mathrm{~cm})$ along the peritoneal fold of both USL during abdominal supracervical hysterectomy, and evaluated the effect on symptoms of UI (Figure 1).

\section{Patients and Methods}

Women with prolapse of the uterus and UI who consulted the Division of Obstetrics and Gynecology of our general hospital (EVK Dortmund) between 2003 and 2007 were candidates for the study.

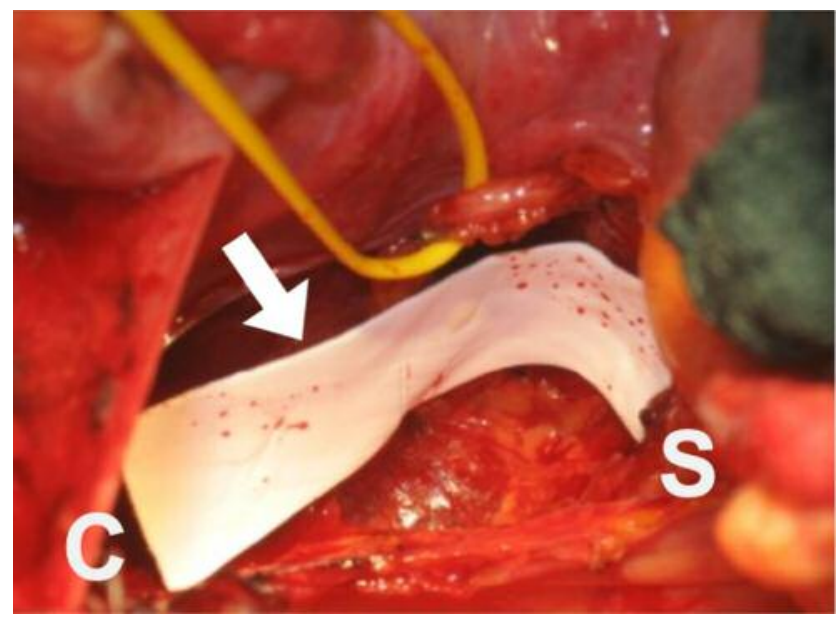

Figure 2. Polytetrafluoroethylene tape (uterosacral ligaments tape) (arrow) Placed between the cervical stump $(C)$ and the sacral vertebra $(S)$ in front of level S2. The right ureter is held up with a yellow elastic band.

The study was approved by the local Ethical Committee and the Clinical Directors' Conference (EL13-2007). Written consent was obtained from all patients. The patients were informed about the Integral Theory and the likelihood of re-establishing urinary continence in addition to apical restoration.

The classification of prolapse of the uterus was according to the Baden-Walker system (12). The classification was conducted with the patient on the gynecological chair and also performed in the standing position before and after each surgical treatment.

The diagnosis of UI was based on subjective complaints of the patients. When necessary, urodynamic measurements were made accordingly. All patients completed validated UI questionnaires before and after each surgical procedure (13). SUI was diagnosed when the patient reported urinary leakage with coughing, sneezing, or physical activity; UUI was defined as involuntary leakage of urine without physical activity or before reaching the toilet in time. Urinary continence was defined as no involuntary loss of urine. Mixed urinary incontinence (MUI) was defined as stress and urgency urinary incontinence.

Outcome measures were the restoration of apical fixation (Baden-Walker system grade 0) and restitution of urinary continence.

All patients underwent apical suspension with bilateral USL replacement. Lower medial laparotomy (Pfannenstiel incision) was performed. As a first step, a supracervical hysterectomy was performed, ensuring that the remaining cervical stump had a length of 2-3 cm. The peritoneum over the USL was incised on both sides of the small pelvis. According to randomisation, polytetrafluoroethylene tapes (ePTFE, GORE-TEX Soft Tissue Patch; W. L. Gore \& Associates, Inc., AZ, USA) were cut into a width of $1 \mathrm{~cm}$ and allocated lengths of 9,10 , and $11 \mathrm{~cm}$. These polytetrafluoroethylene tapes were placed between the cervical stump and the sacral vertebra in front of S2 (Figure 2). These were sutured to the left and right prevertebral fascial layer with two non-resorbable sutures (Figure 3). Additionally, these tapes were sutured to the left and right lateral cut surfaces of the cervical stump (Figure 4). Thereafter, the peritoneum 


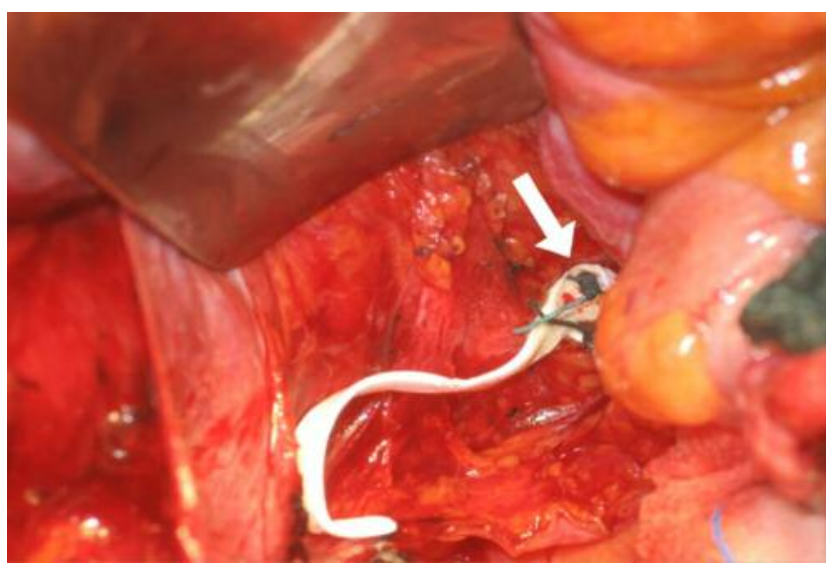

Figure 3. The new uterosacral ligament (polytetrafluoroethylene) tapes were sutured to the left and right (shown here) at the prevertebral fascial layer with two non-resorbable sutures (arrow).

over the cervical stump and along the both USLs was closed using a running suture (Figure 5). The abdomen was closed according to institutional standards.

A TOT was placed according to the technique of Delorme et al. (4).

The patients were examined before and 2, 4, 8, and 16 weeks after surgery. Subjective and objective outcomes were determined at 16 weeks after surgery.

Data collection was performed using Excel sheets (Excel 2007; Microsoft Corporation, Redmond, WA, USA). Metric variables are expressed as means and medians, and frequencies in percentages. Statistical analyses were conducted using GraphPad PRISM Version 4 (GraphPad Software, La Jolla, CA, USA).

\section{Results}

The present study included 31 patients with a median age of 57 years. All women suffered from UI and apical prolapse, with a descent of the uterus until at least half way to the hymenal ring (grade $\geq 1$ ). In Table I, the baseline characteristics are presented. All patients were offered pessary use and most of them had undergone pelvic floor muscle training, as well as conservative treatment of their UI.

After apical suspension, in all $31(100 \%)$ patients, apical vaginal support was restored, that is, apical prolapse was graded as 0 .

All 31 patients reported involuntary loss of urine before surgery; of these patients, six (19\%) and $25(81 \%)$ had SUI and MUI, respectively. After replacement of the USL and PUL (apical suspension and TOT), 18 out of the 31 patients $(58 \%)$ reported urinary continence with no involuntary loss of urine.

The urinary continence rates differed between the patients receiving different lengths of tapes for USL replacement (Table II). The highest continence rate was observed in seven $(78 \%)$ out of nine patients who received the 9-cm-long tapes,

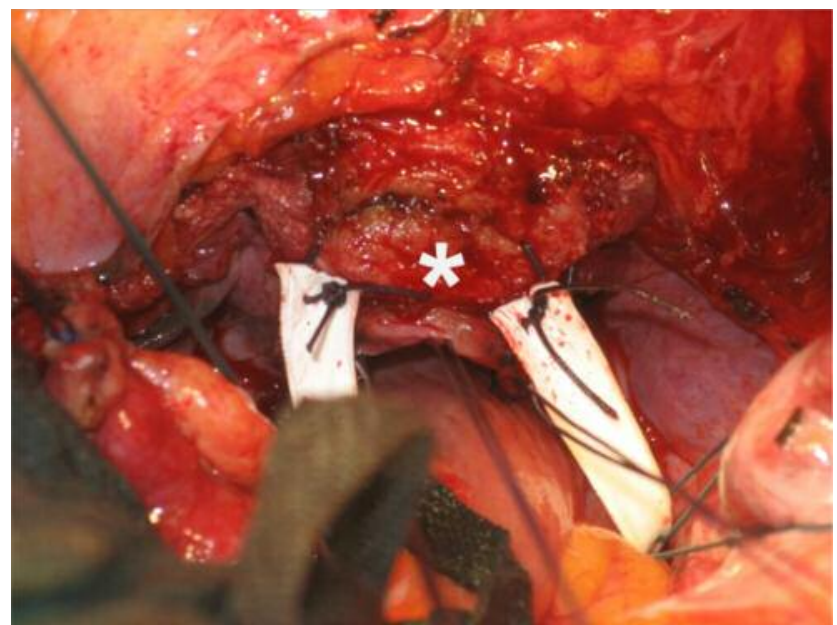

Figure 4. The uterosacral ligament (polytetrafluoroethylene) tapes were sutured to the left and right lateral cut surface of the cervical stump (white asterisk) with non-resorbable sutures.

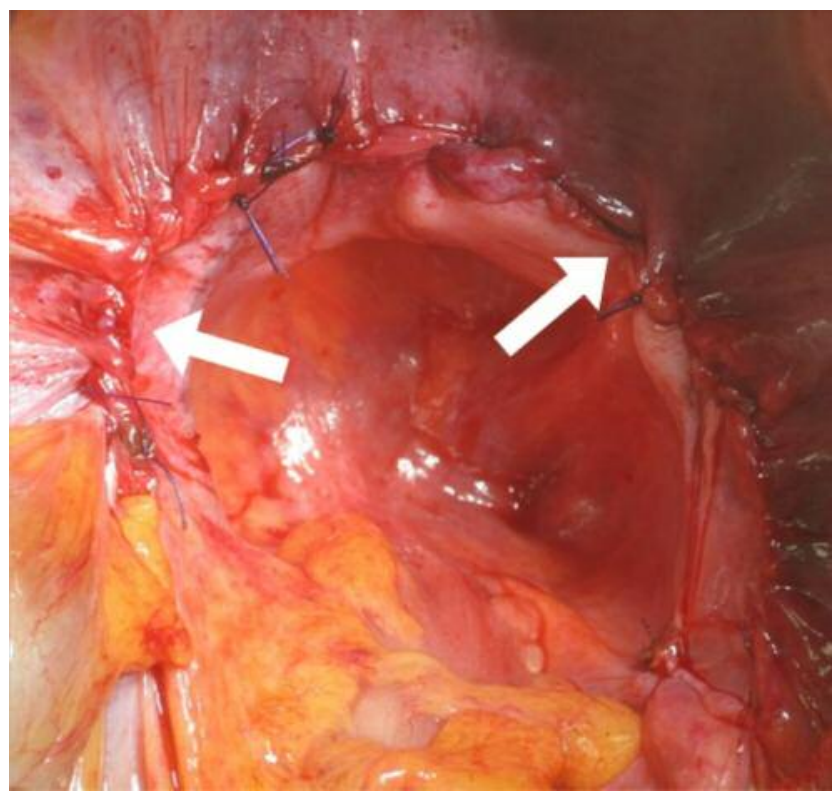

Figure 5. The peritoneum over the cervical stump and along both uterosacral ligaments was closed using a running suture (arrows).

compared to those of $10 \mathrm{~cm}$ and $11 \mathrm{~cm}$ (continence rates $36 \%$ and $64 \%$, respectively). Two patients reported persistent UI symptoms after surgery.

No intraoperative major complications such as vessel or ureter injuries occurred and no mesh erosions were detected within follow-up. Postoperatively, two patients had obstructed defecation and one patient had a urinary tract infection. 


\section{Discussion}

The reported treatments and their results in 31 patients are to be considered as case reports and are therefore not sufficient to draw final conclusions. However, the results observed in these patients warrant further discussion and investigation.

We decided to offer bilateral USL replacement as one alternative method for prolapse surgery (apical fixation). This surgical method was combined with a TOT to re-establish urinary continence according to the Integral Theory (1).

According to the literature, approximately $30 \%$ of patients with pelvic organ prolapse and UI became continent after prolapse surgery (14). That was observed in patients with SUI as well as UUI. However, it was not possible to determine which part of the surgical procedure led to continence. It can be assumed that this is caused by the lack of standardisation of the techniques. For example, every surgeon decides where to place alloplastic materials and sutures, how tight sacrocolpopexies or colporrhaphies are 'tensioned', and whether and how much vaginal tissue needs to be resected.

Considering that the bony dimensions of the small female pelvis are nearly identical among women of different ethnicities, surgical standardisation of bilateral USL replacement is possible (15-17). Basically, in obstetrical textbooks, the obstetric diameters of the small pelvis are given with only small confidence intervals, in the range of few millimetres. Therefore, we decided to 'standardise' our apical fixation and all patients received tapes with defined lengths, irrespective of their body shape or weight. This meant we were able to analyse the effect of an intact apical fixation, in particular the USL, on UI in more detail. Before this analysis, two aspects of the study limitations should be considered, firstly, the wide range of grade 1 apical prolapse, and secondly, the limited number of patients receiving tapes of different lengths.

All patients had varying stages of genital prolapse. During the examination, in the standing position several patients had a higher grade of prolapse (according to the Baden-Walker system) than during the examination on the gynaecological chair. This was predominantly seen in the patients with grade 1 prolapse. Grade 1 in particular covers a wide range of degree of descent of the vaginal apex. We therefore decided to perform vaginal examinations in patients with prolapse or UI as usual on the gynaecological chair but also in the standing position.

Because of the differing lengths of the USL reported in the literature, we were not aware of the correct physiological length of the USL. Based on our own measurements during abdominal hysterectomies and in accordance with the literature, we arrived at the conclusion that the length of the implanted tapes should be between 9 and $11 \mathrm{~cm}$.
Table I. Baseline characteristics of the 31 patients in the study.

\begin{tabular}{lcc}
\hline Characteristic & & Value \\
\hline Age, years & Mean (range) & $57(41-78)$ \\
BMI, kg/m ${ }^{2}$ & Mean (range) & $27(19-37)$ \\
Parity & Mean (range) & $2(0-4)$ \\
Urinary incontinence, & Stress urinary & $6(19 \%)$ \\
n $(\%)$ & incontinence (SUI) & \\
& Mixed urinary & $25(81 \%)$ \\
Apical prolapse*, & incontinence (MUI) & $0(0 \%)$ \\
n $(\%)$ & Grade 0 & $13(42 \%)$ \\
& Grade 1 & $18(58 \%)$ \\
\hline
\end{tabular}

BMI: Body mass index. *Apical prolapse according to the BadenWalker system (9).

Table II. Clinical data and outcome of the 31 patients, divided according to length (tensioning) of alloplastic uterosacral ligament (USL) replacement.

\begin{tabular}{|c|c|c|c|c|c|}
\hline \multirow{2}{*}{$\begin{array}{l}\text { Length } \\
\text { of USL } \\
\text { tape }\end{array}$} & \multirow{2}{*}{$\begin{array}{c}\text { MUI } \\
(\mathrm{UUI}), \\
\mathrm{n}(\%)\end{array}$} & \multirow[t]{2}{*}{$\begin{array}{l}\text { Grade of } \\
\text { prolapse }\end{array}$} & \multirow{2}{*}{$\begin{array}{c}\text { Mean age } \\
\text { (range), } \\
\text { years }\end{array}$} & \multicolumn{2}{|c|}{$\begin{array}{c}\text { Status after } \\
\text { AS+TOT, n (\%) }\end{array}$} \\
\hline & & & & Continent & Incontinent \\
\hline $9 \mathrm{~cm}$ & $9(9)$ & II-III & $55(49-76)$ & $7(78 \%)$ & $2(22 \%)$ \\
\hline $10 \mathrm{~cm}$ & $11(9)$ & I-IV & $57(41-78)$ & $7(64 \%)$ & $4(36 \%)$ \\
\hline $11 \mathrm{~cm}$ & $11(7)$ & I-IV & $60(55-75)$ & $4(36 \%)$ & $7(64 \%)$ \\
\hline Total & 31 & - & - & $18(58 \%)$ & $13(42 \%)$ \\
\hline
\end{tabular}

MUI: Mixed (urgency and stress) urinary incontinence; UUI: urgency urinary incontinence; AS: apical suspension; TOT: transobturator tape; USL: uterosacral ligament.

The incontinence rates were different, especially between the group with 11-cm-long tapes and the remaining groups. In the group with 11-cm-long tapes, urinary continence was only achieved in three patients, who had increased voiding frequency in addition to SUI. The highest cure rate of UI was observed in patients with 9-cm-long tapes. Moreover, seven out of nine patients with prior MUI became continent.

These case reports indicated that the replacement of the USL and PUL led to continence in $18(58 \%)$ out of the 31 patients. Therefore, the predictions of Integral theory must be tested further.

It is tempting to speculate that shorter tapes might further improve the outcome, namely continence rate. However, shorter tapes might narrow the entrance into the small pelvis, which might lead to defecation problems. Furthermore, in our measurements during hysterectomies, we obtained the impression that extremely shorter tapes $(e . g .6 \mathrm{~cm})$ are not physiological. To evaluate the precise role of the USL in continence, future treatment studies should randomise separate placement of USL tapes from that of PUL tapes (TOT) (18). 
Finally, these case reports indicated that it was possible to re-establish urinary continence in patients with SUI and UUI by the replacement of the USL and PUL, as predicted in the Integral Theory. The use of identical tapes and defined surgical techniques (standardisation) will enable the analysis of clinical outcomes and other parameters of interest between different treatment centres. This would enable the improvement of current and future treatment of UI.

\section{Conclusion}

According to the Integral Theory, SUI and UUI in women result from the same anatomical defect, namely the laxity of the anterior vaginal wall which supports the vesicourethral junction (3). Bilateral elevation and defined tensioning (standardisation) of the vagina led to urinary continence.

\section{Conflicts of Interest}

The Authors report no conflicts of interest in regard to this study.

\section{Authors' Contributions}

SL: Article writing, project development, and data editing. SG: Article writing, statistical analysis. PM: project development, article writing. WJ: performing surgeries, article writing, project development, and data collection.

\section{Acknowledgements}

The Authors thank Mrs. Elke Neuman for documentation of all the data and keeping contact with the patients.

\section{References}

1 Petros PE and Ulmsten UI: An integral theory and its method for the diagnosis and management of female urinary incontinence. Scand J Urol Nephrol Suppl 153: 1-93, 1993. PMID: 8108659.

2 Ulmsten U and Petros P: Surgery for female urinary incontinence. Curr Opin Obstet Gynecol 4(3): 456-462, 1992. PMID: 1623156.

3 Petros PE and Ulmsten U: Bladder instability in women: A premature activation of the micturition reflex. Neurourol Urodyn 12(3): 235-239, 1993. PMID: 8330046.

4 Delorme E: Transobturator urethral suspension: Mini-invasive procedure in the treatment of stress urinary incontinence in women. Prog Urol 11(6): 1306-1313, 2001. PMID: 11859672.

5 Petros P: Creating a gold standard surgical device: Scientific discoveries leading to tvt and beyond: Ulf ulmsten memorial lecture 2014. Int Urogynecol J 26(4): 471-476, 2015. PMID: 25693655. DOI: 10.1007/s00192-015-2639-3

6 Amundsen CL, Flynn BJ and Webster GD: Anatomical correction of vaginal vault prolapse by uterosacral ligament fixation in women who also require a pubovaginal sling. J Urol 169(5): 1770-1774, 2003. PMID: 12686830. DOI: 10.1097/ 01.ju.0000061472.94183.26
7 Barber MD, Visco AG, Weidner AC, Amundsen CL and Bump RC: Bilateral uterosacral ligament vaginal vault suspension with site-specific endopelvic fascia defect repair for treatment of pelvic organ prolapse. Am J Obstet Gynecol 183(6): 1402-1410; discussion 1410-1401, 2000. PMID: 11120503. DOI: 10.1067/ mob.2000.111298

8 Maher CF, Baessler KK, Barber MD, Cheong C, Consten ECJ, Cooper KG, Deffieux X, Dietz V, Gutman RE, van Iersel JJ, Nager CW, Sung VW and de Tayrac R: Surgical management of pelvic organ prolapse. Climacteric 22(3): 229-235, 2019. PMID: 30572743. DOI: $10.1080 / 13697137.2018 .1551348$

9 DeLancey JO: The anatomy of the pelvic floor. Curr Opin Obstet Gynecol 6(4): 313-316, 1994. PMID: 7742491.

10 Ramanah R, Berger MB, Parratte BM and DeLancey JO: Anatomy and histology of apical support: A literature review concerning cardinal and uterosacral ligaments. Int Urogynecol J 23(11): 14831494, 2012. PMID: 22618209. DOI: 10.1007/s00192-012-1819-7

$11 \mathrm{Vu}$ D, Haylen BT, Tse K and Farnsworth A: Surgical anatomy of the uterosacral ligament. Int Urogynecol J 21(9): 1123-1128, 2010. PMID: 20458468. DOI: 10.1007/s00192-010-1147-8

12 Baden WF and Walker TA: Genesis of the vaginal profile: A correlated classification of vaginal relaxation. Clin Obstet Gynecol 15(4): 1048-1054, 1972. PMID: 4649139, DOI: 10.1097/00003081-197212000-00020

13 Avery K, Donovan J, Peters TJ, Shaw C, Gotoh M and Abrams P: Iciq: A brief and robust measure for evaluating the symptoms and impact of urinary incontinence. Neurourol Urodyn 23(4): 322-330, 2004. PMID: 15227649. DOI: 10.1002/nau.20041

14 de Boer TA, Salvatore S, Cardozo L, Chapple C, Kelleher C, van Kerrebroeck P, Kirby MG, Koelbl H, Espuna-Pons M, Milsom I, Tubaro A, Wagg A and Vierhout ME: Pelvic organ prolapse and overactive bladder. Neurourol Urodyn 29(1): 30-39, 2010. PMID: 20025017. DOI: 10.1002/nau.20858

15 Rizk DE, Czechowski J and Ekelund L: Dynamic assessment of pelvic floor and bony pelvis morphologic condition with the use of magnetic resonance imaging in a multiethnic, nulliparous, and healthy female population. Am J Obstet Gynecol 191(1): 83-89, 2004. PMID: 15295346. DOI: 10.1016/j.ajog.2003.12.041

16 Chen L, Ramanah R, Hsu Y, Ashton-Miller JA and Delancey JO: Cardinal and deep uterosacral ligament lines of action: MRI based 3D technique development and preliminary findings in normal women. Int Urogynecol J 24(1): 37-45, 2013. PMID: 22618207. DOI: 10.1007/s00192-012-1801-4

17 Grob ATM, Olde Heuvel J, Futterer JJ, Massop D, Veenstra van Nieuwenhoven AL, Simonis FFJ and van der Vaart $\mathrm{CH}$ : Underestimation of pelvic organ prolapse in the supine straining position, based on magnetic resonance imaging findings. Int Urogynecol J 30(11): 1939-1944, 2019. PMID: 30656361. DOI: 10.1007/s00192-018-03862-0

18 Ludwig S, Becker I, Mallmann P and Jager W: Comparison of solifenacin and bilateral apical fixation in the treatment of mixed and urgency urinary incontinence in women: Urge 1 study, a randomized clinical trial. In Vivo 33(6): 1949-1957, 2019. PMID: 31662524. DOI: 10.21873/invivo.11690

Received January 9, 2020

Revised January 23, 2020

Accepted January 28, 2020 